\title{
Persistence of the valence bond glass state in the double perovskites $\mathrm{Ba}_{2-x} \mathrm{Sr}_{x} \mathrm{YMoO}_{6}$
}

\author{
A. C. Mclaughlin* \\ Department of Chemistry, University of Aberdeen, Meston Walk, Aberdeen AB24 3UE, United Kingdom \\ M. A. de Vries \\ School of Physics and Astronomy, E.C. Stoner Laboratory, University of Leeds, Leeds LS2 9JT, United Kingdom \\ J.-W. G. Bos ${ }^{\dagger}$ \\ Department of Chemistry, EPS, Heriot-Watt University, Edinburgh EH14 4AS, United Kingdom \\ (Received 30 September 2009; revised manuscript received 2 August 2010; published 14 September 2010)
}

\begin{abstract}
The $\mathrm{Ba}_{2-x} \mathrm{Sr}_{x} \mathrm{YMoO}_{6}$ double perovskites with a geometrically frustrated face centered $\mathrm{Mo}^{5+}\left(s=\frac{1}{2}\right)$ sublattice have been investigated using neutron powder-diffraction, magnetic-susceptibility, and heat-capacity measurements. Reduction in the average $A$-site radius results in the distortion of the crystal structure from cubic for $x=0$ to monoclinic for $x=2$ without affecting the Y/Mo inversion $(<1 \%)$. Magnetic-susceptibility measurements evidence two paramagnetic regimes for all samples. The high-temperature (HT) Curie constants are independent of composition $x\left[0.26(1) \mathrm{emu} \mathrm{mol}^{-1} \mathrm{~K}^{-1}\right]$ while the low-temperature (LT) value gradually increases from $0.04(1)$ emu mol $\mathrm{K}^{-1} \mathrm{~K}^{-1}$ for $x=0$ to $-0.10(1)$ emu $\mathrm{mol}^{-1} \mathrm{~K}^{-1}$. The HT Weiss temperature $(\theta)$ increases from $-159 \mathrm{~K}(x=0)$ to $-50 \mathrm{~K}(x=2)$ while the LT values are $-2.3(3) \mathrm{K}$ for $x<2$ and $+10 \mathrm{~K}$ for $x=2$. Neutron powder diffraction does not evidence any Neel ordering. The $x=2$ sample is weakly ferromagnetic below $8 \mathrm{~K}\left(M_{\text {sat }} \sim 0.1 \mu_{\mathrm{B}} /\right.$ Mo in $1 \mathrm{~T}$ at $\left.5 \mathrm{~K}\right)$ which is consistent with the small positive LT Weiss temperature. The magnetic data are consistent with a valence bond glass state where the bulk of the spins condense into spin singlets as previously observed for $\mathrm{Ba}_{2} \mathrm{YMoO}_{6}$, leaving a small fraction of the spins isolated or only weak ferromagnetically coupled to each other.
\end{abstract}

DOI: 10.1103/PhysRevB.82.094424

PACS number(s): 75.50.Dd, 75.30.Cr, 61.66.Fn, 61.05.F-

\section{INTRODUCTION}

The study of magnetic frustration is an important area of research in contemporary condensed-matter physics. ${ }^{1-3}$ Geometric frustration arises when antiferromagnetically coupled spins are situated in lattices based on triangles or tetrahedra. This results in the destabilization of long-range-ordered "Neel-type" ground states, and instead exotic magnetic grounds states, such as spin liquids, spin glasses, and spin ices may be observed. ${ }^{1-3}$ Double perovskite materials with composition $A_{2} M M^{\prime} \mathrm{O}_{6}$ contain two interpenetrating $M$ and $M^{\prime}$ face-centered lattices, which are built from edge sharing $M\left(M^{\prime}\right)$ tetrahedra. These materials are therefore susceptible to geometric frustration. ${ }^{4}$ However, in most cases the nearest-neighbor frustration is relieved by next-nearestneighbor interactions and long-range Neel order is observed. $^{5-7}$ Exceptions occur for systems with small spin quantum number, primarily $s=\frac{1}{2}$ systems. Notable examples include the $\mathrm{Sr}_{2} \mathrm{MReO}_{6}(\mathrm{M}=\mathrm{Mg}$ and $\mathrm{Ca})$ double perovskites that show spin-glass behavior. ${ }^{8,9}$ We have recently reported unconventional magnetism in $\mathrm{Ba}_{2} \mathrm{YMoO}_{6}$, where no magnetic order is observed down to $2 \mathrm{~K}$, while the Weiss temperature is $\sim-160 \mathrm{~K} .{ }^{10}$ The experimental results show that as the temperature is reduced there is a gradual freezing of spins into a disordered pattern of spin singlets so that $\mathrm{Ba}_{2} \mathrm{YMoO}_{6}$ is the first example of an exotic valence bond glass (VBG), which is a cooperative spin-singlet ground state. The VBG phase had been previously proposed theoretically (Ref. 11) and is characterized by an amorphous arrangement of dimers and absence of magnetic ordering. There is no gap in the electronic and spin density of states although a pseudogap is evidenced. Independently, Aharen et $a l .{ }^{12}$ reported on the magnetic properties of $\mathrm{Ba}_{2} \mathrm{YMoO}_{6}$ and their observations support our conclusions. In contrast to this unconventional magnetism, Mo double perovskites containing the earlier lanthanides $(M=\mathrm{Nd}, \mathrm{Sm}$, and $\mathrm{Eu})$ have been found to show long-range Neel order with $T_{N}=15 \mathrm{~K}(\mathrm{Nd})$, $130 \mathrm{~K}(\mathrm{Sm})$, and $112 \mathrm{~K}(\mathrm{Eu}){ }^{13-15}$ These compounds are also Jahn-Teller active with a singly occupied $4 d t_{2 g}$ orbital in the octahedrally coordinated $\mathrm{Mo}^{5+}$. There is no evidence of magnetic order for $\mathrm{Ba}_{2} M \mathrm{MoO}_{6}$ with $M=\mathrm{Gd}$, Dy, Y, Yb, and Er. ${ }^{13}$

Here, we present an investigation of the structural and magnetic properties of the new series $\mathrm{Ba}_{2-x} \mathrm{Sr}_{x} \mathrm{YMoO}_{6}$ with $x=0,0.5,1,1.5$, and 2 . This follows from our detailed investigation of the $x=0$ composition, including ac-susceptibility,

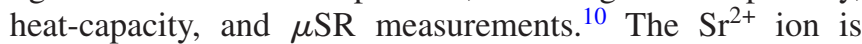
smaller than $\mathrm{Ba}^{2+}$, leading to the Glazer tilting of the $\mathrm{MoO}_{6}$ octahedra, and lowering the symmetry from cubic $(x \leq 0.5)$ to monoclinic $\left(P 2_{1} / n\right)$ for $x=2$. In this paper we show that the octahedral tilting does not destabilize the VBG state and that the low-temperature (LT) susceptibilities, which are dominated by a minority fraction of free $s=\frac{1}{2}$ spins, are observed to change from paramagnetic for $x<2$ to ferromagnetic for $x=2$.

\section{EXPERIMENTAL}

$\mathrm{Ba}_{2-x} \mathrm{Sr}_{x} \mathrm{YMoO}_{6}(x=0,0.5,1,1.5$, and 2$)$ were prepared by the solid-state reaction of stoichiometric oxides of $\mathrm{Y}_{2} \mathrm{O}_{3}$, $\mathrm{MoO}_{3}, \mathrm{BaCO}_{3}$, and $\mathrm{SrCO}_{3}$ powders of at least $99.99 \%$ purity. These were ground, die pressed into a pellet, and heated under flowing $5 \% \mathrm{H}_{2} / \mathrm{N}_{2}$. The final synthesis temperature 
was $1200-1250{ }^{\circ} \mathrm{C}$ with three intermediate regrinding steps to ensure phase homogeneity. It was found that a first heating step of $\sim 2 \mathrm{~h}$ at $900{ }^{\circ} \mathrm{C}$ in air, and thorough homogenization helps to prevent the formation of $(\mathrm{Ba} / \mathrm{Sr}) \mathrm{MoO}_{4}$ and $\mathrm{Y}_{2} \mathrm{O}_{3}$ impurities. Phase purity was confirmed by laboratory $\mathrm{X}$-ray powder diffraction using a Bruker D8 Advance diffractometer.

Neutron-diffraction patterns of $\mathrm{Ba}_{2} \mathrm{YMoO}_{6}$ (2 $\mathrm{g}$ sample) and $\mathrm{Sr}_{2} \mathrm{YMoO}_{6}$ (5 g sample) were recorded at a wavelength of $1.5943 \AA$ on instrument SuperD2B at the ILL, Grenoble. The samples were contained in vanadium cans and neutrondiffraction patterns were recorded with an acquisition time of $1 \mathrm{~h}$ at several temperatures between 4 and $290 \mathrm{~K}$ for $\mathrm{Sr}_{2} \mathrm{YMoO}_{6}$ and for $2 \mathrm{~h}$ at $4 \mathrm{~K}$ and RT for $\mathrm{Ba}_{2} \mathrm{YMoO}_{6}$. Lowtemperature neutron-diffraction patterns were recorded on the high intensity beamline D20 at a wavelength of $2.42 \AA$ in order to evidence magnetic ordering of the $\mathrm{Mo}^{5+}$ spins.

The temperature and field dependences of the magnetization were measured using a Quantum Design magnetic property measurement system (MPMS) superconducting quantum interference device (SQUID) magnetometer. The heat capacities of $\mathrm{Sr}_{2} \mathrm{YMoO}_{6}$ and $\mathrm{Sr}_{2} \mathrm{YNbO}_{6}$ were measured using the heat-capacity option of the Quantum Design physical property measurement system (PPMS). These measurements were done on thin platelets of about $6 \mathrm{mg}$. The sample coupling constant was always larger than $95 \%$.

\section{RESULTS}

\section{A. Crystal structures}

The neutron powder-diffraction patterns of $\mathrm{Ba}_{2} \mathrm{YMoO}_{6}$ and $\mathrm{Sr}_{2} \mathrm{YMoO}_{6}$ were fitted by the Rietveld method ${ }^{16}$ using the GSAS program. ${ }^{17}$ The backgrounds were fitted using linear interpolation and the peak shapes were modeled using a pseudo-Voigt function. Our data confirmed the ideal cubic double perovskite structure [space group (SG) $F m \overline{3} m$ ] for $x$ $=0$ reported previously by Cussen et al. ${ }^{13}$ Rietveld refinement confirmed the nominal stoichiometry and the absence of Y/Mo cation inversion $(<1 \%)$. No structural phase transitions were observed upon cooling and the $4 \mathrm{~K}$ data set was successfully fitted using the $F m \overline{3} m$ structural model [Fig. $\mathrm{S} 1(\mathrm{a})] .{ }^{18}$ The refined lattice constants, atomic parameters, selected bond lengths, and the fits statistics are reported in Table S1.

For $\mathrm{Sr}_{2} \mathrm{YMoO}_{6}$ an excellent fit to the monoclinic space group $P 2_{1} / n$ is observed at all temperatures [Table S2, Figure $\mathrm{S} 1(\mathrm{~b})]$. There is no evidence of oxygen nonstoichiometry or significant antisite disorder and the $\mathrm{Mo}^{5+}$ and $\mathrm{Y}^{3+}$ site occupancies refine to 1.01(1) and 0.98 (1), respectively, at 290 K. Figures 1(a) and 1(b) show the temperature variation in the lattice constants and cell volume in $\mathrm{Sr}_{2} \mathrm{YMoO}_{6}$. A normal thermal expansion of both $a$ and $c$ are observed but $b$ is observed to increase as the temperature decreases. The monoclinic angle increases from $90.222(2)^{\circ}$ to $90.315(2)^{\circ}$ as the temperature is reduced to $4 \mathrm{~K}$. There are no significant discontinuities in Mo-O bond lengths in this temperature interval (Fig. 2); Mo-O(1) and Mo-O(3) are observed to decrease and increase gradually upon cooling whereas there is
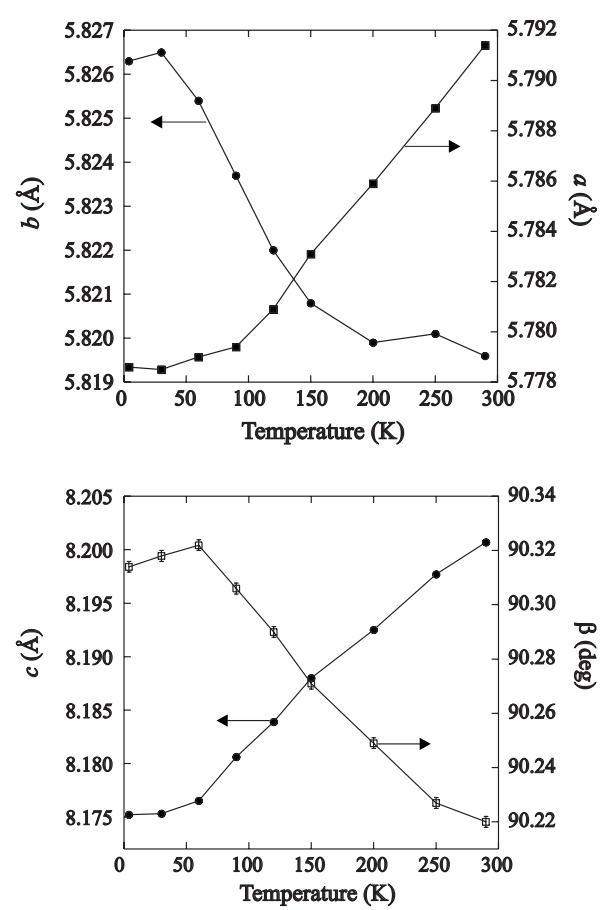

FIG. 1. (a) The temperature variation in $a$ and $b$ cell parameters for $\mathrm{Sr}_{2} \mathrm{YMoO}_{6}$ evidencing an increase in $b$ below $200 \mathrm{~K}$ and (b) the evolution of $c$ cell parameter and monoclinic angle.

no change in $\mathrm{Mo}-\mathrm{O}(2)$. The $\mathrm{O}-\mathrm{M}-\mathrm{O}$ bond angles are almost $90^{\circ}$ at all temperatures (Table S1) and there is no evidence of a discontinuity in the Y-O-Mo tilt angles (Fig. S2).

There was no evidence of magnetic peaks in neutrondiffraction data recorded at $2 \mathrm{~K}$ for both $x=0$ and $x=2$ on the high-intensity beamline D20 (Fig. S3). Results from SQUID magnetometry discussed in the next section reveal a small ferromagnetic (FM) moment of only $0.12 \mu_{\mathrm{B}}$ below $8 \mathrm{~K}$ for $x=2$. If the sublattice magnetization were of comparable order it would be below the detection limit at D20, of approximately $0.2 \mu_{\mathrm{B}}$.

The room-temperature structures of the $x=0.5, x=1$, and $x=1.5$ compositions were studied using laboratory $\mathrm{x}$-ray powder diffraction. The $x=0.5$ diffraction pattern did not show any additional reflections and was therefore fitted using the $F m \overline{3} m$ structural model (Table I). For $x=1$, additional

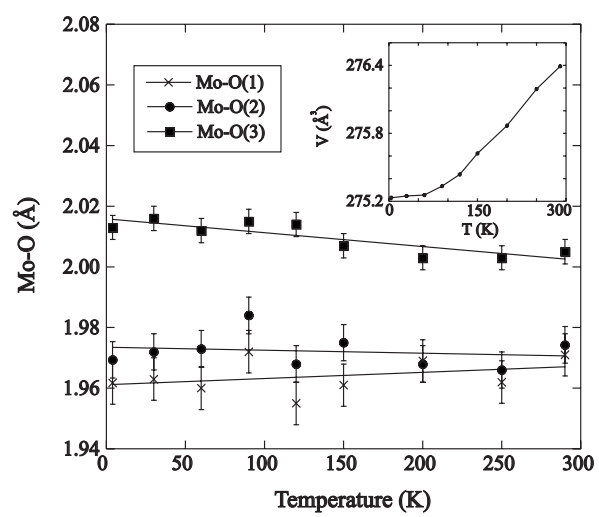

FIG. 2. Variation in Mo-O bond lengths with temperature. The inset shows the temperature evolution of the cell volume. 
TABLE I. Lattice constants from laboratory powder x-ray diffraction, Curie constants, and Weiss temperatures obtained from Curie-Weiss fits to magnetic-susceptibility data for the $\mathrm{Ba}_{2-x} \mathrm{Sr}_{x} \mathrm{YmoO}_{6}$ double perovskites.

\begin{tabular}{|c|c|c|c|c|c|}
\hline & $X=0$ & $x=0.5$ & $x=1$ & $x=1.5$ & $x=2$ \\
\hline SG & $F m \overline{3} m$ & $F m \overline{3} m$ & $I 4 / m$ & $P 2_{1} / n$ & $P 2_{1} / n$ \\
\hline$a$ axis $(\AA)$ & $8.3892(1)$ & $8.3629(1)$ & $5.8875(1)$ & $5.8530(1)$ & $5.7959(1)$ \\
\hline$b$ axis $(\AA)$ & & & & $5.8386(1)$ & $5.8240(1)$ \\
\hline$c$ axis $(\AA)$ & & & $8.2928(2)$ & $8.2456(1)$ & $8.2059(2)$ \\
\hline$\beta(\operatorname{deg})$ & & & & $89.88(1)$ & $90.23(1)$ \\
\hline Vol $\left(\AA^{3}\right)$ & $590.41(2)$ & $584.80(2)$ & $287.45(2)$ & $281.78(2)$ & $277.00(2)$ \\
\hline$C\left(\mathrm{emu} \mathrm{mol}^{-1} \mathrm{~K}^{-1}\right)^{\mathrm{a}}$ & $0.27(1)$ & $0.26(1)$ & $0.26(1)$ & $0.24(1)$ & $0.26(1)$ \\
\hline$\theta(\mathrm{K})^{\mathrm{a}}$ & $-159(5)$ & $-158(3)$ & $-116(2)$ & $-98(3)$ & $-50(2)$ \\
\hline$C\left(\mathrm{emu} \mathrm{mol} \mathrm{m}^{-1} \mathrm{~K}^{-1}\right)^{\mathrm{b}}$ & $0.04(1)$ & $0.07(1)$ & $0.08(1)$ & $0.09(1)$ & $0.10(1)$ \\
\hline$\theta(\mathrm{K})^{\mathrm{b}}$ & $-2.3(3)$ & $-2.8(3)$ & $-2.3(3)$ & $-2.1(3)$ & $+10(1)$ \\
\hline
\end{tabular}

Between 2-30 K.

${ }^{\mathrm{b}}$ Between 200-300 K.

weak reflections are observed that obey the $h+k+l=2 n$ reflection condition, and the pattern was fitted using the $I 4 / \mathrm{m}$ superstructure as reported for $\mathrm{Ba}_{2} \mathrm{NdMoO}_{6}$ (Table I). ${ }^{13}$ For $x=1.5$, further weak reflections are observed, breaking the $h+k+l=2 n$ reflection condition, and this pattern was fitted using the $P 2_{1} / n$ superstructure (Table I), as found for the $x$ $=2$ end member.

\section{B. Magnetization}

The valence bond glass state has previously been characterized in $\mathrm{Ba}_{2} \mathrm{YMoO}_{6}$ by $\mathrm{dc}$ and ac magnetometry, heatcapacity, and $\mu \mathrm{SR}$ measurements. ${ }^{10}$ The dc magnetometry results showed two Curie-Weiss $(\mathrm{CW})$ regions with a reduction in the Curie constant $(C)$ at low temperature. The results are consistent with the majority of spins becoming bound into spin-singlet valence bonds as the temperature is decreased. The small paramagnetic moment observed at low temperature is due to weakly coupled spins which are left isolated in a disordered pattern of spin-singlet valence bonds pairing neighboring spins. The inverse zero-field-cooled (ZFC) magnetic susceptibilities $[1 / \chi(T)]$ of the $\mathrm{Ba}_{2-x} \mathrm{Sr}_{x} \mathrm{YMoO}_{6}$ double perovskites are shown in Fig. 3. For all samples CW behavior is discernible in two different temperature regimes. CW fits between 200-300 K yield Curie constants $(C)$ of $\sim 0.26(1)$ emu $\mathrm{mol}^{-1} \mathrm{~K}^{-1}$ and Weiss temperatures $(\theta)$ from $-160 \mathrm{~K}\left(x=0\right.$ and $\left.x=\frac{1}{2}\right)$ to $-50 \mathrm{~K}(x=2$, Table I). The experimental Curie constant corresponds to an effective moment of $1.45 \mu_{\mathrm{B}}$, reduced compared to the spinonly value of $1.7 \mu_{\mathrm{B}}$ for $\mathrm{Mo}^{5+}\left(s=\frac{1}{2}\right)$. The increase in the Weiss temperature from -160 to $-50 \mathrm{~K}$ (Table I) indicates that the net antiferromagnetic (AF) interactions between $\mathrm{Mo}^{5+}$ spins become weaker (or competing FM interactions become stronger) as $x$ increases in the $\mathrm{Ba}_{2-x} \mathrm{Sr}_{x} \mathrm{YMoO}_{6}$ series. In the low-temperature regime $(2-30 \mathrm{~K})$ a considerable reduction in the high-temperature (HT) constants $C$ and $\theta$ is evident, as previously reported for $\mathrm{Ba}_{2} \mathrm{YMoO}_{6} \cdot{ }^{10}$ In this temperature range the Curie constants are found to increase from $0.04(1) \mathrm{emu} / \mathrm{mol}$ for $x=0$ to $0.09(1) \mathrm{emu} / \mathrm{mol}$ for $x=1.5$
(Table I). The data was fitted above the FM transition for $x$ $=2$ (between 15 and $30 \mathrm{~K}$ ) yielding $\theta=+10 \mathrm{~K}$ and the Curie constant, $C=0.10(1) \mathrm{emu} / \mathrm{mol}$ (Table I). These values are up to $1 / 3$ of the high-temperature Curie constant and too large to ascribe to a paramagnetic impurity phase.

There is no evidence of magnetic order for $x=0-1.5$. For $x=2$ a ferromagnetic transition is observed at low temperature. As shown in Fig. 4, there is a steep rise in the magnetic susceptibility of $\mathrm{Sr}_{2} \mathrm{YMoO}_{6}$, characteristic of $\mathrm{FM}$ ordering transition. The Curie temperature was estimated at $8 \mathrm{~K}$ by extrapolating the maximum $(-d M / d T)$ slope to zero magnetization. A hysteresis loop was recorded at $5 \mathrm{~K}$ between $\pm 1 \mathrm{~T}$, evidencing a small remnant moment of $0.05 \mu_{\mathrm{B}}$ and a coercive field of $\sim 100$ Oe [Fig. 4 (inset)]. The magnetization is almost saturated at $1 \mathrm{~T}\left(M_{\mathrm{sat}}=0.12 \mu_{\mathrm{B}}\right)$ which is much smaller than expected for $\mathrm{Mo}^{5+}\left(4 d^{1}\right)$. The FM state for $x=2$ is confirmed by an Arrott plot [Fig. 4 (inset)]. Theory predicts that the (high-field) isotherms of $M^{2}$ vs $H / M$ are parallel lines for ferromagnets and that isotherms below $T_{\text {c }}$ have a negative $H / M$-axis intersect. ${ }^{19}$ This is clearly evident at $5 \mathrm{~K}$, confirming the FM state. The majority of nonmetallic double perovskites are AF and the observation of a $\mathrm{FM}$ transition in $\mathrm{Sr}_{2} \mathrm{YMoO}_{6}$ is therefore surprising.

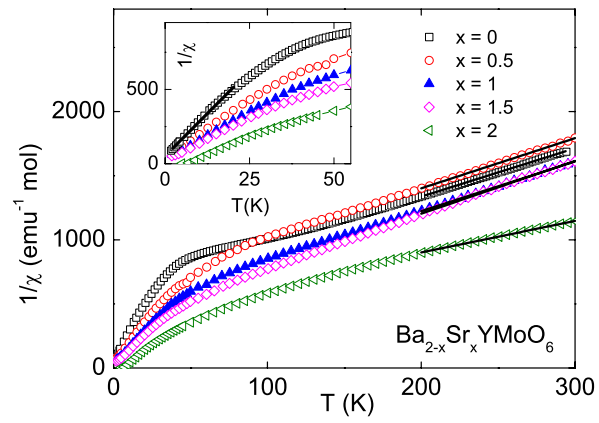

FIG. 3. (Color online) Temperature dependence of the ZFC inverse magnetic susceptibility $[1 / \chi(T)]$ for $\mathrm{Ba}_{2-x} \mathrm{Sr}_{x} \mathrm{YMoO}_{6}$ solid solutions recorded in a field of $1 \mathrm{~T}$. The inset shows the expanded low-temperature region between 0-60 K. 


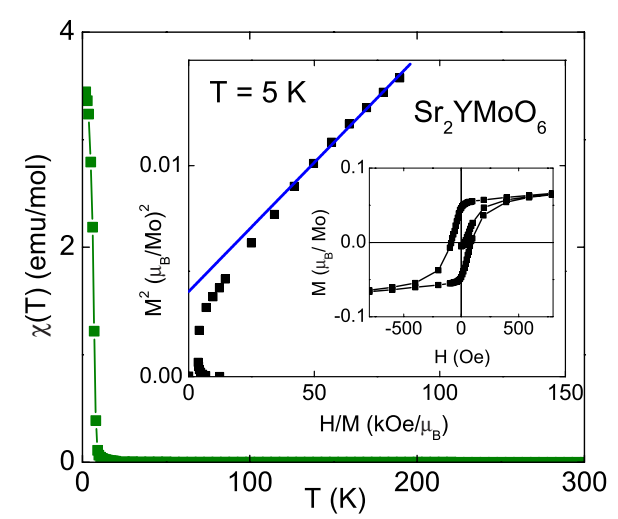

FIG. 4. (Color online) Temperature dependence of the ZFC magnetic susceptibility $\chi(T)$ for $\mathrm{Sr}_{2} \mathrm{YMoO}_{6}$ evidencing a ferromagnetic transition below $8 \mathrm{~K}$. The inset shows the hysteresis loop recorded at $5 \mathrm{~K}$ and corresponding Arrott plot.

\section{Heat capacity}

Heat-capacity measurements on $\mathrm{Ba}_{2} \mathrm{YMoO}_{6}$ indicate that the magnetic entropy is gradually released over a wide range of temperatures below $\sim 150 \mathrm{~K}$ (VBG transition temperature) with a broad maximum around $50 \mathrm{~K} .{ }^{10}$ This gradual freezing and crossover region around $50 \mathrm{~K}$ is consistent with the pseudogap predicted for the VBG. ${ }^{11}$ The temperature dependence of the heat capacity divided by temperature $(C / T$ $=d S / d T)$ for $\mathrm{Sr}_{2} \mathrm{YMoO}_{6}$ is shown in Fig. 5. A clear difference between $x=2$ and the diamagnetic reference sample $\mathrm{Sr}_{2} \mathrm{YNbO}_{6}$ is apparent below $\sim 150 \mathrm{~K}$. The residual heat capacity $\left(C_{\text {magn }} / T\right)$ is shown in the inset and takes the shape of a broad hump with a transition at $8 \mathrm{~K}$ superposed. The hightemperature behavior is comparable to that for the $x=0$ (Ref. 10) compound but at low temperature there is an additional feature corresponding to the ferromagnetic ordering. The absence of a clear anomaly implies that this is still a glassy state but one where the isolated spin moments left out in the disordered singlet dimerization experience a net ferromagnetic exchange leading to a small ferromagnetic moment. $\mu \mathrm{SR}$ experiments are currently underway to further investigate this unconventional ferromagnetic state in more detail. The total recovered entropy is $8.8 \pm 1.3 \mathrm{~J} \mathrm{~mol}^{-1} \mathrm{~K}^{-1}$, where

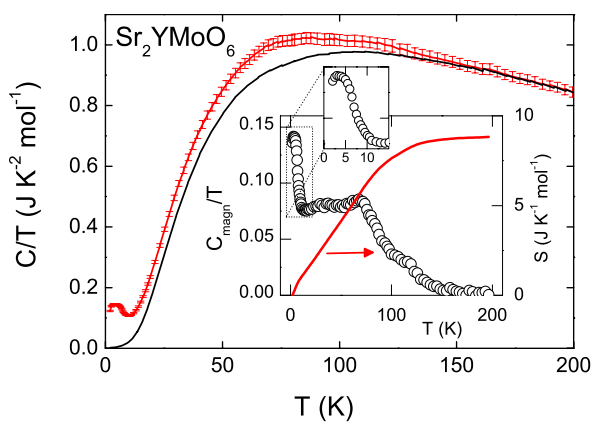

FIG. 5. (Color online) Temperature variation in the heat capacity of $\mathrm{Sr}_{2} \mathrm{YMoO}_{6}$ evidencing a ferromagnetic transition at $8 \mathrm{~K}$; top and bottom data show $C / T$ for $\mathrm{Sr}_{2} \mathrm{YMoO}_{6}$ and diamagnetic analog $\mathrm{Sr}_{2} \mathrm{YNbO}_{6}$, respectively. The inset shows the magnetic heat capacity and the total entropy released as a function of temperature. the standard deviation reflects the larger inaccuracy of the measurement at higher temperatures. This value is in rough agreement with a fourfold degenerate $J=3 / 2$ ground state $(R \ln 4=11.5)$. The bulk of the magnetic entropy $(\sim 90 \%)$ is recovered above $10 \mathrm{~K}$, indicating that the FM transition only involves a small fraction of the present spins. For both $x=0$ and $x=2$ the heat capacity does not become zero at the lowest temperature measured which is consistent with an ungapped spectrum and a small residual entropy as expected for the VBG.

\section{DISCUSSION}

Results from Rietveld refinement of neutron data show no evidence for an orbital ordering (OO) transition in the $x=0$ and $x=2$ materials. This contrasts with $\mathrm{Ba}_{2} M \mathrm{MoO}_{6}(M$ $=\mathrm{Nd}$ and $\mathrm{Sm}$ ) where $\mathrm{OO}$ has been observed using neutron powder diffraction. ${ }^{13,15}$ The slightly different Mo-O bond lengths (Fig. 2) are common in monoclinic double perovskites and cannot directly be interpreted as resulting from an OO. [In principle, a transition above $300 \mathrm{~K}$ is possible but this appears unlikely as $T_{\mathrm{OO}} \quad\left(=T_{\mathrm{N}}\right)=130 \mathrm{~K}$ for $\mathrm{Ba}_{2} \mathrm{SmMoO}_{6}{ }^{15}$ ] The replacement of $\mathrm{Ba}^{2+}$ by the smaller ion $\mathrm{Sr}^{2+}$ results in increased octahedral tilting in the double perovskite structure and a lowering of the crystal symmetry from $F m \overline{3} m$ for $x=0$ to $P 2_{1} / n$ for $x=2$. The Glazer tilt systems for these space groups are $a^{0} a^{0} a^{0}$ and $a^{-} a^{-} b^{+}$, respectively, ${ }^{20}$ with average tilt angles $\phi=(180$ $-\langle\mathrm{Mo}-\mathrm{O}-\mathrm{Y}\rangle) / 2]=0$ for $x=0^{\circ}$ and $11.9(2)^{\circ}$ for $x=2$ at room temperature. As $x$ increases $>0.5$ in $\mathrm{Ba}_{2-x} \mathrm{Sr}_{x} \mathrm{YMoO}_{6}$, the HT Weiss temperature $\theta$ rises from -158 to $-50 \mathrm{~K}$. The Weiss temperature reflects the average magnetic interaction and suggests that FM (potential) superexchange is enhanced with octahedral tilting. This is in agreement with the appearance of ferromagnetism for the most distorted $\mathrm{Sr}_{2} \mathrm{YMoO}_{6}$ and it is plausible that the ferromagnetic exchange takes place along the Mo-O-Y-O-Mo pathways. The LT Weiss temperatures remain close to zero for $x<2$, which reflects the weak magnetic interaction between the remaining free $s=\frac{1}{2}$ spins. The LT behavior of the $x=2$ sample is different but consistent with the increased importance of FM further-neighbor interactions. Magnetic susceptibility and magnetization measurements reveal a FM alignment of the remaining spins below 8 $\mathrm{K}$ with a small saturation moment of $\sim 0.12 \mu_{\mathrm{B}} /$ Mo. The LT Weiss temperature for $x=2$ is $+10 \mathrm{~K}$, consistent with ferromagnetic interactions between the unpaired spins that remain below the spin-singlet dimerization temperature $(\sim 150 \mathrm{~K})$ and in good agreement with the FM "transition" temperature of $8 \mathrm{~K}$. The HT Curie constants (Table I) do not depend on $x$ and hence, are not affected by the structural distortions. The LT Curie constants, in contrast, increase from $0.04 \mathrm{emu} \mathrm{mol}^{-1} \mathrm{~K}^{-1}$ for $x=0$ to $0.10 \mathrm{emu} \mathrm{mol}^{-1} \mathrm{~K}^{-1}$ for $x$ $=2$. This suggests that more unpaired $s=\frac{1}{2}$ spins remain upon cooling.

The heat-capacity measurements provide further evidence in favor of a VBG state in the $x=2$ material. The magnetic contribution is characterized by a broad hump with a peak at the FM transition superposed. The shape is reminiscent of that reported for $x=0$ and is consistent with the gradual 
freezing of free $s=\frac{1}{2}$ spins into singlet bonds. The heat capacity also reveals that the onset temperature of the VBG state is $\sim 150 \mathrm{~K}$. This is similar to the value for $x=0$, which confirms that the near-neighbor AF exchange strength along the Mo-O-O-Mo pathway is not significantly affected by the structural distortions.

Recently ferromagnetism with small saturation $\left(\sim 0.2 \mu_{\mathrm{B}}\right)$ and paramagnetic moments has been reported for $\mathrm{Ba}_{2} \mathrm{NaOsO}_{6}\left(C=0.045\right.$ emu mol K${ }^{-1}$, corresponding to $12 \%$ of the expected value for $s=\frac{1}{2}$ spins). ${ }^{21-23}$ These small values have been proposed to result from a single-ion effect for the $t_{2 g}^{1}$ configuration, where the orbital and spin contributions to the magnetic moment cancel out. ${ }^{23}$ In the current case, the larger HT Curie constants $\left(C=0.27 \mathrm{emu} \mathrm{mol}^{-1} \mathrm{~K}^{-1}\right)$ suggest that single-ion effects are not as important. Moreover, as indicated by Aharen et al., ${ }^{13}$ the single-ion energy scale for $\mathrm{Mo}^{5+}$ ions is $\sim 1500 \mathrm{~K}$ and therefore more than an order of magnitude larger than the magnetic transition occurring in these materials. Hence, while effects related to spin-orbit coupling are undoubtedly important, the reduction in $C$ at low temperature is the result of a gradual freezing of the spins into a disordered pattern of spin singlets in the $\mathrm{Ba}_{2-x} \mathrm{Sr}_{x} \mathrm{YMoO}_{6}$ series.

In summary, we have studied the magnetic and structural properties of $\mathrm{Ba}_{2-x} \mathrm{Sr}_{x} \mathrm{YMoO}_{6}$. As $x$ increases from 0 to 2, the crystal symmetry changes from cubic $(F m \overline{3} m)$ to monoclinic $\left(P 2_{1} / n\right)$ due to increased Glazer tilting. This also reduces the symmetry of the $\mathrm{Mo}^{5+}\left(s=\frac{1}{2}\right)$ face-centered lattice. We have shown that the VBG state previously reported for $x=0$ is robust against these distortions and by inference robust against a change in sign of the second-neighbor interactions (although with an increasing number of free spins at low temperature, as the symmetry is reduced). This implies that the VBG state is not solely an effect of the frustration arising from a delicate balance of near-neighbor and furtherneighbor interactions and that the orbital degrees of freedom are likely to play a crucial role. Variable-temperature neutron powder-diffraction results on $\mathrm{Ba}_{2} \mathrm{YMoO}_{6}$ and $\mathrm{Sr}_{2} \mathrm{YMoO}_{6}$ reveal the absence of an $\mathrm{OO}$ transition, which contrasts with the magnetically ordered $\mathrm{Mo}^{5+}\left(s=\frac{1}{2}\right)$ double perovskites where Neel and OO order appear coupled. ${ }^{15}$

\section{ACKNOWLEDGMENTS}

We thank the Leverhulme Trust (ACM), EPSRC for provision of beamtime at the ILL, and P. Henry for assistance with the neutron experiments. J.-W.G.B. acknowledges R. J. Cava for scientific discussions.

\footnotetext{
*a.c.mclaughlin@abdn.ac.uk

†j.w.g.bos@hw.ac.uk

${ }^{1}$ A. P. Ramirez, Annu. Rev. Mater. Sci. 24, 453 (1994).

${ }^{2}$ J. E. Greedan, J. Mater. Chem. 11, 37 (2001).

${ }^{3}$ L. Balents, Nature (London) 464, 199 (2010).

${ }^{4}$ H. Karunadasa, Q. Huang, B. G. Ueland, P. Schiffer, and R. J. Cava, Proc. Natl. Acad. Sci. U.S.A. 100, 8097 (2003).

${ }^{5}$ P. D. Battle and W. J. Macklin, J. Solid State Chem. 52, 138 (1984).

${ }^{6}$ Jan-Willem G. Bos and J. P. Attfield, Phys. Rev. B 70, 174434 (2004).

${ }^{7}$ S. J. Makowski, J. A. Rodgers, P. F. Henry, J. P. Attfield, and J.-W. G. Bos, Chem. Mater. 21, 264 (2009).

${ }^{8}$ C. R. Wiebe, J. E. Greedan, P. P. Kyriakou, G. M. Luke, J. S. Gardner, A. Fukaya, I. M. Gat-Malureanu, P. L. Russo, A. T. Savici, and Y. J. Uemura, Phys. Rev. B 68, 134410 (2003).

${ }^{9}$ C. R. Wiebe, J. E. Greedan, G. M. Luke, and J. S. Gardner, Phys. Rev. B 65, 144413 (2002).

${ }^{10}$ M. A. de Vries, A. C. Mclaughlin, and J.-W. G. Bos, Phys. Rev. Lett. 104, 177202 (2010).

${ }^{11}$ M. Tarzia and G. Biroli, EPL 82, 67008 (2008).

${ }^{12}$ T. Aharen, J. E. Greedan, C. A. Bridges, A. A. Aczel, J. Rod-
}

riguez, G. MacDougall, G. M. Luke, T. Imai, V. K. Michaelis, S. Kroeker, H. Zhou, C. R. Wiebe, and L. M. D. Cranswick, Phys. Rev. B 81, 224409 (2010).

${ }^{13}$ E. J. Cussen, D. R. Lynham, and J. Rogers, Chem. Mater. 18, 2855 (2006).

${ }^{14}$ A. C. McLaughlin, Solid State Commun. 137, 354 (2006).

${ }^{15}$ A. C. Mclaughlin, Phys. Rev. B 78, 132404 (2008).

${ }^{16}$ H. M. Rietveld, Acta Crystallogr. 22, 151 (1967).

${ }^{17}$ A. C. Larson and R. B. Von Dreele, Los Alamos National Laboratory Report No. LA-UR-86-748, 1994 (unpublished).

${ }^{18}$ See supplementary material at http://link.aps.org/supplemental/ 10.1103/PhysRevB.82.094424 for results of the structural refinements.

${ }^{19}$ A. Arrott, Phys. Rev. 108, 1394 (1957).

${ }^{20}$ P. M. Woodward, Acta Crystallogr., Sect. B: Struct. Sci. 53, 32 (1997).

${ }^{21}$ K. E. Stitzer, M. D. Smith, and H. C. zur Loye, Solid State Sci. 4, 311 (2002).

${ }^{22}$ A. S. Erickson, S. Misra, G. J. Miller, R. R. Gupta, Z. Schlesinger, W. A. Harrison, J. M. Kim, and I. R. Fisher, Phys. Rev. Lett. 99, 016404 (2007).

${ }^{23}$ K.-W. Lee and W. E. Pickett, EPL 80, 37008 (2007). 